\title{
Self-Start Performance Evaluation in Darrieus-Type Vertical Axis Wind Turbines: Methodology and Computational Tool Applied to Symmetrical Airfoils
}

\author{
N.C. Batista ${ }^{1}$, R. Melício ${ }^{1}$, J.C.O. Matias ${ }^{1,2}$ and J.P.S. Catalão ${ }^{1,3}$ \\ ${ }^{1}$ University of Beira Interior, ${ }^{2}$ Centre for Aerospace Science and Technologies \\ R. Fonte do Lameiro, 6200-001 Covilhã (Portugal) \\ Phone: +351275 329 914, Fax: +351275 329972 \\ e-mail: nelson.batista@gmail.com, rui.melicio@di.ubi.pt, matias@ubi.pt \\ ${ }^{3}$ Center for Innovation in Electrical and Energy Engineering, Instituto Superior Técnico \\ Av. Rovisco Pais 1, 1049-001 Lisbon (Portugal) \\ Phone: +351218417 000, Fax: +351218499242 \\ e-mail: catalao@ubi.pt
}

\begin{abstract}
An increased interest in vertical axis wind turbines has been stimulated by the rapid growth of wind power generation and by the need for a smarter electrical grid with a decentralized energy generation, especially in the urban areas. The lift type vertical axis wind turbines (Darrieus wind turbine) performance prediction is a very complex task, since its blades move around the rotor axis in a three dimensional aerodynamic environment that lead to several flow phenomena, such as, dynamic stall, flow separation, flow wake deformations and their natural inability to self-start. These issues can be overcome with the use of several more or less complex solutions, being one of them the development of a blade profile capable of making the wind turbines self-start. This paper focuses on presenting a new methodology for fast development of new blade profiles, for self-start capable Darrieus wind turbines, which is a complex and time-consuming task.
\end{abstract}

\section{Key words}

Vertical axis wind turbine, blade profile, self-start, pressure coefficient.

\section{Introduction}

The increasing cost of fossil fuels and the different agreements among the industrialized countries with the aim of reducing $\mathrm{CO}_{2}$ emissions has driven the renewable sources in an increased acceptance for energy production.

The wind energy systems have been considered as one of the most cost effective of all the currently exploited renewable sources, so the demand and investment in wind energy systems has increased in the last decade.

Several studies have been conducted to model, simulate [1] and characterize [2] the wind behaviour to stimulate the acceptance of the wind energy in the market, by offering tools to help and ease the enterprise I\&D.

The investment in wind energy for the 27 EU Member States is expected to grow in the next 20 years reaching almost $€ 20$ billion in 2030 , with $60 \%$ of that investment in offshore systems [3]. In the past for Portugal alone, the wind power goal foreseen for 2010 was established by the government as $3750 \mathrm{MW}$.
The value of $3750 \mathrm{MW}$ already represented about $25 \%$ of the total installed capacity [4]. Nevertheless, this value has recently been raised to $5100 \mathrm{MW}$, by the most recent governmental goals for the wind sector.

As the penetration level of wind power increases into the power systems, the overall performance of the electric grid will increasingly be affected by the characteristics of wind turbines. One of the major concerns related to the high penetration level of the wind turbines is the impact on power system stability and power quality [5]

The decentralized energy generation is an important solution in a smarter electrical grid with a growing acceptance for the urban areas. Also, the increasing need for more environmentally sustainable housing and the new European norms regulating this issue, have contributed for the promotion of wind energy systems in buildings.

In urban areas the wind is very turbulent and unstable with fast changes in direction and velocity. In these environments the vertical axis wind turbines (VAWT) have several advantages over horizontal axis wind turbines (HAWT). Several solutions have been presented to overcome the Darrieus type VAWT inability to selfstart: use of a guide-vane [6], using a hybrid configuration of a Savonius VAWT (drag type wind turbine) and a Darrieus VAWT (lift type wind turbine) [7]-[9], use of mechanical system to optimize the blade pitch [10], [11], use of blades that change their form during operation [12], [13], or a specific blade profile capable of offering self-start capabilities to the wind turbine without extra components [14], [15].

Our paper presents a methodology and computational tool for fast development of a specific blade profile capable of offering self-start capabilities to the VAWT without extra components.

The VAWT in order to self-start relying only on the blades profile, without the help of extra components and external power, must take advantage of the drag forces caused by the wind on the blades when the turbine is in a stopped position, without compromising the wind turbine performance at high tip speed ratio (TSR). 
If possible the lift forces should be used in cooperation with the drag forces to induce the self-start capabilities of the wind turbine, especially when the turbine is stopped and the wind flow starts to achieve higher velocities.

So, it is essential to study the blade profile behaviour in relation to the wind when the wind turbine is stopped. One difficulty arises here, since the blade may be at any given position around the rotor, implying the need to study the blade profile at any angular position from $0^{\circ}$ to $360^{\circ}$. In the previous situation, the dynamic stall behaviour, air flow separation and any other aerodynamic disturbances must be taken in consideration [16], [17].

To study these aerodynamic disturbances, requires a high computational processing time, which leads to a time consuming situation not advisable in the first steps of the development studies. Hence, the methodology that is proposed for the development of new blades profile is only suitable for fast analysis when there is the need to compare several blade profiles solutions to start restricting and eliminating different designs. It is important not to forget the analysis of the different aspects of the wind flow disturbances acting on the wind turbine in a more advanced stage of the study.

This paper is organized as follows. Section II presents the methodology and computational tool used for the development of the self-start profile capabilities of the new blade profile analysis. Section III presents the output data for several known blade profiles. Finally, concluding remarks are given in Section IV.

\section{Methodology and Computational Tool}

To study a blade design performance, capable of offering the VAWT the ability to self-start, there is the need to study the wind flow behaviour around the airfoil when the wind turbine is in a stopped position. In this situation, the wind flow may encounter the blade profile at any given azimuthal position from $0^{\circ}$ to $360^{\circ}$.

Accordingly, the dynamic stall behaviour, air flow separation and any other aerodynamic disturbances must be taken in consideration [14]-[18]. To study these aerodynamic disturbances, requires a high computational processing time, which leads to a time consuming situation not advisable in the first steps of the development studies.

So, as mentioned previously, the methodology that is present here to demonstrate the development of new VAWT blade profiles is only suitable for fast analysis when there is the need to compare several blade profile solutions to start restricting and eliminating different designs. The proposed methodology is also useful when associated to a blade design software application, giving a fast view on the blade performance when any modification is made to the profile design. Moreover, it is very important not to forget the analysis of the different aspects of the wind flow disturbances acting on the wind turbine.

\section{A. Methodology}

To study the blade profile design modifications and the implications that those modifications bring to the wind turbine performance, a close relation between the surface of the blade and the wind flow must be created. In this methodology the pressure coefficient $C_{p r}$ is going to be used, which is a dimensionless number that describes the relative pressure throughout a flow field and is intimately correlated to the flow velocity, and can be calculated at any point of the flow field.

To study the $C_{p r}$ around the blade profile surface, first there is the need to divided it into segments as shown in Figure 1, and then to calculate the $C_{p r}$ in each segment.

The smaller the segments the more accurate will be the resulting analysis.

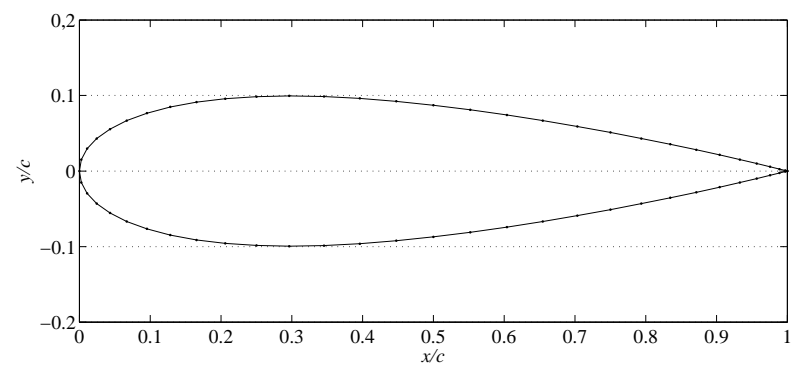

Fig. 1. Segmented NACA0020 blade profile.

The relation between the blade profile segment and the $C_{p r}$ is shown in Figure 2.

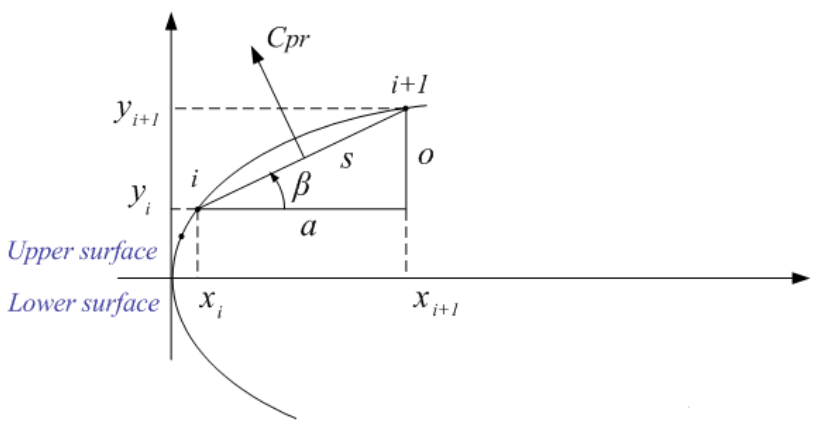

Fig. 2. Pressure coefficient $C_{p r}$ acting on the blade profile surface.

Figure 2 shows the points $i$ and $i+1$ of the segment of length $s$ in the blade profile surface and their corresponding Cartesian coordinates in the $x$ and $y$ axis. In the triangle formed by segment $s$ in relation to the $x$ and $y$ axis, $o$ represents the opposite side and $a$ represents the adjacent side and their length are given by

$$
\begin{aligned}
& a=x_{i+1}-x_{i} \\
& \begin{cases}o=y_{i+1}-y_{i} & \text { upper surface } \\
o=y_{i}-y_{i+1} & \text { lower surface }\end{cases}
\end{aligned}
$$


When $o$ is positive it means that the surface segment is oriented in the direction to the wind turbine rotation, while when $o$ is negative the segment is oriented in the opposite direction.

The blade profile segment length $s$ is given by

$s=\sqrt{a^{2}+|o|^{2}}$

The segment angle $\beta$ in relation to the blade chord line (the $x$ axis) is given by

$\beta=\arctan (o / a)$

By having the $C_{p r}$ exerted in each segment of the blade, there is the need to get the $C_{p r}$ contribution to the tangential force $T_{p r}$ and the $C_{p r}$ contribution to the normal force $N_{p r}$, which are shown in Fig. 3.

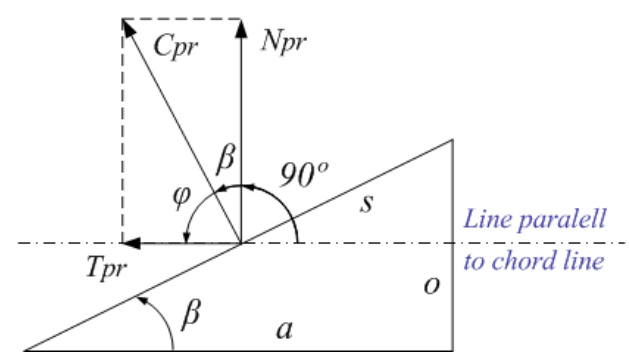

Fig. 3. Pressure coefficient $C_{p r}$ and its contribution to the tangential force $T_{p r}$ and normal force $N_{p r}$ acting on the blade profile surface.

As shown in Figure 3, the angle $\varphi$ is the $C_{p r}$ angle in relation to the blade chord line and is given by

$\varphi=108^{\circ}-90^{\circ}-\beta$

The $C_{p r}$ contribution to the tangential force $T_{p r}$ and to the normal force $N_{p r}$ can be expressed as in (6) and (7).

These contributions must be multiplied by the blade profile segment length $s$ and are given by

$$
\begin{gathered}
\begin{cases}T_{p r}=C_{p r} \cos (\varphi) s & \text { when } o \geq 0 \\
T_{p r}=-C_{p r} \cos (\varphi) s & \text { when } o<0\end{cases} \\
\begin{cases}N_{p r}=C_{p r} \sin (\varphi) s & \text { upper surface } \\
N_{p r}=-C_{p r} \sin (\varphi) s & \text { lower surface }\end{cases}
\end{gathered}
$$

The equations (6) and (7) show the relation between the $C_{p r}, T_{p r}, N_{p r}$, the angle $\varphi$ and the segment length $s$.

\section{B. Computational Tool}

The aerodynamic behaviour and performance data for different blade profiles is not always available, and in the majority of the cases is incomplete. This data is very hard to obtain and it is a very time-consuming task. Several Computational Fluid Dynamics (CFD) tools are commonly used to generate the aerodynamic performance data needed.

The computational tool presented in this paper is the JavaFoil [19], which is a fast processing computational tool with a validated good accuracy.

This tool is able to analyse different blade profiles of any configuration, offering all kind of different output data, for instance: velocity and pressure coefficient distribution along the blade chord; lift, drag and momentum coefficient; flow field with pressure vectors; flow stream lines and pressure distribution along the fluid flow; boundary layer evaluation cards; polar card evaluation with the relations between lift and drag coefficients and with the angle of attack variation; and other information.

The JavaFoil is also able to evaluate multi-foil and ground effect configurations.

One important feature of JavaFoil is the ability to save all the analysis and output data to a file. Adding the last feature to its integrated scripting module, it is possible to automate the computational processes and to complement the information needs with other tools.

In the theoretical background of the tool, it uses several methods for airfoil analysis, mainly divided in two main areas

1) Potential Flow Analysis: which is done by a panel method with a linear varying vorticity distribution based in the XFOIL code. This method is used to calculate the velocity distribution along the surface of the airfoil

2) Boundary Layer Analysis: which analyses the upper and lower surfaces of the airfoil with different equations, starting with the panel method and performing several calculations based in [20], in a called integral boundary layer method.

Depending on the Reynolds number and other parameters, the tool gives us the ability to choose different analysis methods and configurations offering more flexibility to the computational processing.

\section{Using the Methodology and the Computational Tool}

To obtain the $C_{p r}$ vectors along the blade profile surface with the JavaFoil tool, after uploading the profile coordinates, in the velocity area the maximum and minimum angle and its increment must be set. 
By selecting the pressure coefficient parameter for evaluation and analysing the airfoil, it is possible to save the processed data to a file with the $x$ and $y$ coordinates and the corresponding $C_{p r}$ vectors, needed for the method explained in the previous section of this paper.

In a normal operation of a VAWT, the variations of pressure and wind speed have little influence in the wind density, so the wind flow can be treated as being incompressible.

Hence, it this assumed that: when $C_{p r}$ at one point is null the pressure at that point is the same as the pressure of the undisturbed wind flow; when $C_{p r}$ is equal to one, that point is a stagnation point, meaning that the flow velocity at that point is null (relevant when optimizing the drag forces); when $C_{p r}$ is negative in the point of study, the wind is moving at a higher speed than in the undisturbed wind flow (relevant when optimizing the lift forces).

When studying the self-start capabilities of a VAWT, it is very important to study the drag effects exerted in the turbine blades.

By getting the $C_{p r}$ with values between one and null, it is possible to see the blade profile segments that are suffering from drag forces.

By relating these values with the negative or positive values of $o$, it is possible to see the drag forces contribution to the forward movement of the blades or to the opposite direction, respectively.

\section{Airfoil Data Evaluation}

For the airfoils data evaluation by applying the methodology and computational tool presented in previous sections, the symmetrical NACA where selected, namely: NACA0012, NACA0018, NACA0020, NACA0025, NACA0030.

The NACA0012 and NACA0018 are the classical blade profiles used in the VAWT. These profiles are considered to have low self-start capabilities. The thicker NACA0020 blade profile can be commonly found in the straight-bladed Darrieus wind turbine. The thicker blades show a better performance of self-start [14].

By evaluating several symmetrical blade profiles with an aerodynamic multi-criteria shape optimization, reference [21] considered the NACA0025 to have an optimal shape design.

The NACA0030 by having a thicker blade profile is closer to a self-start capacity nature. This ticker blade leads to an increase drag at high tip speed ratios leading to a decrease of performance. All five blade profiles are shown in Figure 4.

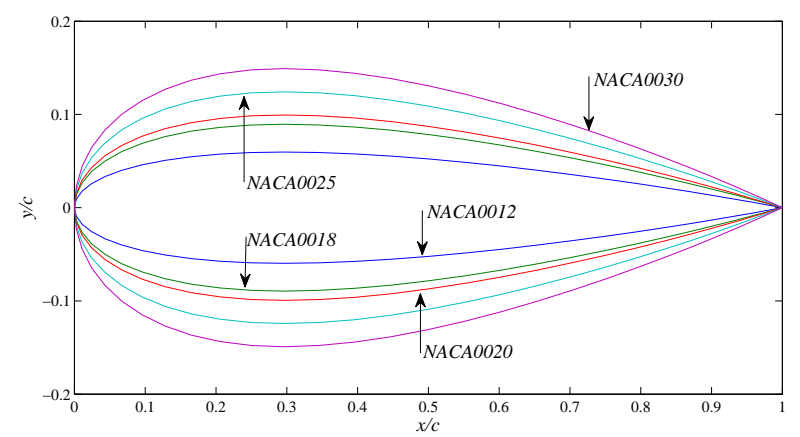

Fig. 4. NACA0012, NACA0018, NACA0020, NACA0025 and NACA0030 blade profiles.

In order to apply the proposed methodology, the pressure coefficient needs to be calculated around the blade profile. For the data evaluation presented here, the $C_{p r}$ is calculated for all segments around the blade profile for any given angle between $0^{\circ}$ and $360^{\circ}$.

The JavaFoil tool offers the pressure coefficient evaluation associated with the $x$ and $y$ coordinates. This evaluation can be automatically done to the entire $360^{\circ}$ at the same time in the velocity area.

By applying the equations (1) and (2) to the given $x$ and $y$ coordinates, the opposite side $o$ and the adjacent side $a$ are obtained. By applying equation (3), the length of the airfoil surface exposed to the wind forces is obtained. With the equations (4) and (5), the $C_{p r}$ angle in relation to the blade chord line $\varphi$ is obtained.

With the data calculated previously, it is now possible to determine the $C_{p r}$ contribution to the tangential force $T_{p r}$ and the $C_{p r}$ contribution to the normal force $N_{p r}$. These forces are related to the actual tangential and normal forces responsible for the blades movement, by the pressure coefficient.

The $C_{p r}$ contribution to the tangential force $T_{p r}$, and the $C_{p r}$ contribution to the normal force $N_{p r}$, for the chosen NACA airfoils are shown in Figures 5 and 6 , respectively.

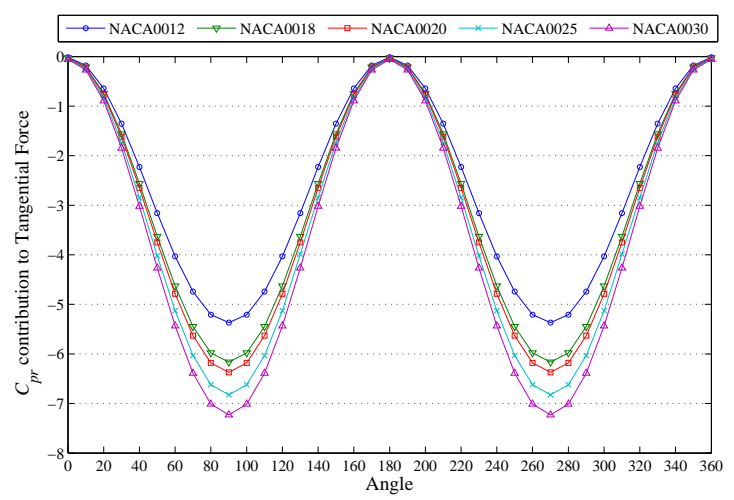

Fig. 5. $C_{p r}$ contribution to the tangential force $T_{p r}$. 


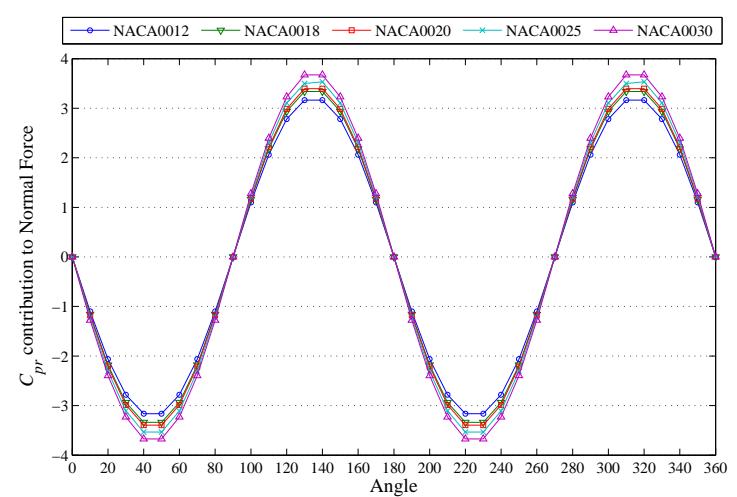

Fig. 6. $C_{p r}$ contribution to the normal force $N_{p r}$.

In Figure 5 it can be seen that the ticker the blade the higher the pressure coefficient contribution to the forward movement of the wind turbine blades (contribution to the tangential force). The NACA0030 present $26 \%$ better performance than NACA0012.

In Figure 6 it can be seen that the airfoil NACA0012 presents the most desirable behavior. The smaller the axial exerted forces the smaller the needed blade/arms connection reinforcements.

When the wind turbine is in a stopped position the drag forces have a considerable contribution to the self-start of the wind turbine. The pressure coefficient is also used to study the drag contribution to the forward movement of the wind turbines blades. In an incompressible flow, when the pressure coefficient reaches values between one and null, that is a stagnation point. The study of these values that contribute to the forward movement of the wind turbine blades are shown in Figure 7.

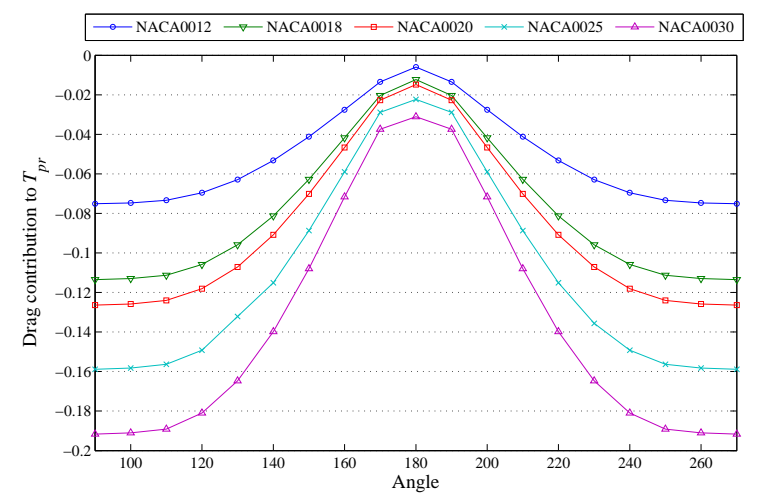

Fig. 7. Drag contribution to the forward movement of the wind turbine blades $T_{p r}$.

In Figure 7 it can be seen that the ticker the blades the higher the drag contribution to the forward movement of the wind turbine blades. In the NACA0025 the drag forces contributing to the tangential force are $110 \%$ higher than in NACA0012, and in the NACA0030 the forces are $150 \%$ higher than in NACA0012.

These data are in good agreement with the studies presented in [14] and [15]. Indeed, the ticker blades are able to provide the wind turbine the self-start capabilities, while the thinner blade wind turbines has most likely unable to self-start.

\section{Conclusion}

This paper focuses on the analysis of the behaviour of the flow around a VAWT blade airfoil when it is in a stopped position, in order to boost the development of new blade profiles capable of offering the lift type VAWT the ability to self-start. A new methodology is introduced in cooperation with the JavaFoil computational tool, to study these phenomena, as a fast approach for comparing the several blade profile design modifications and enhancements in new airfoil developments. To present the data output, several blade profiles were studied and presented in this paper. The NACA0030 airfoil presents the best performance. This blade presents the best $C_{p r}$ contribution to the tangential force and shows the best drag forces contribution to the forward movement of the wind turbine blades. The NACA0012 shows the lowest $C_{p r}$ contribution to the normal force.

\section{References}

[1] R. Melício, V.M.F. Mendes, and J.P.S. Catalão, "Modelling and Simulation of a Wind Energy System with Fractional Controllers", in: ICREPQ'10, vol. 2, paper 261, Granada, Spain, March 2010.

[2] F. S. Correia, L. C. Gonçalves, and L. C. Pires, "Characterization of wind energy potential and availability at Beira Interior (Portugal)", in: ICREPQ'10, vol. 10, paper 686, Granada, Spain, March 2010.

[3] European Wind Energy Association, "The economics of wind energy", EWEA 2009.

[4] A. Estanqueiro, R. Castro, P. Flores, J. Ricardo, M. Pinto, R. Rodrigues, and J. Peças Lopes, "How to prepare a power system for $15 \%$ wind energy penetration: the Portuguese case study", Wind Energy, vol. 11, no. 1, pp. 75-84, Jan.-Feb. 2008.

[5] R. Melício, V.M.F. Mendes, and J.P.S. Catalão, "Fractional-order control and simulation of wind turbines with full-power converters", in: MELECON 2010, pp. 320-325, Valletta, Malta, Apr. 2010.

[6] M. Taka, H. Kuma, T. Maeda, Y. Kamada, M. Oki, and A. Minoda. "A straight-bladed vertical axis wind turbine with a directed guide vane row-effect of guide vane geometry on the performance", J. Therm. Sci. 2009; 18(1):54-57

[7] R. Gupta, A. Biswas, K.K. Sharma. "Comparative study of a three-bucket Savonius rotor with a combined threebucket Savonius-three-bladed Darrieus rotor", Renew. Energy 2008; 33(9):1974-1981.

[8] Md. Jahangir Alam, M.T. Iqbal. "Design and development of hybrid vertical axis turbine", in: CCECE '09, pp. 1178183, St. John's, NL, 3-6 May 2009.

[9] T. Wakui, Y. Tanzawa, T. Hashizume, T. Nagao. "Hybrid configuration of Darrieus and Savonieus rotors for standalone wind turbine-generator systems", IEEJ Trans. Electr. Electron. Eng. 2004; 124-B(2):259-266.

[10] P. Cooper and O. Kennedy. "Development and analysis of a novel vertical axis wind turbine", in: Proc. of 42nd Annual Conf. of the Australian and New Zealand Solar Energy Soc. 2004, Perth, Australia, 1-3 December 2004.

[11] I. Paraschivoiu, O. Trifu, F. Saeed. "H-Darrieus wind turbine with blade pitch control”, Int. J. of Rotating Machinery 2009, 2009:1-7.

[12] J. DeCoste, A. Smith, D. White, D. Berkvens, and J. Crawford, "Self-starting Darrieus wind turbine", Design Project Mech. 4020. Dalhousie University, Hallifax, Canada, April 2004. 
[13] P. Bhatta, M. A. Paluszek, J. B. Mueller, "Individual blade pitch and camber control for vertical axis wind turbines", in: WWEC2008, Kingston, Canada, 24 June 2008.

[14] B. K. Kirke, "Evaluation of self-starting vertical axis wind turbines for stand-alone applications". PhD Thesis, School of Engineering, Griffith University, Australia, April 1998.

[15] R. Dominy, P. Lunt, A. Bickerdyke, and J. Dominy, "Selfstarting capability of a Darrieus turbine", J. of Power and Energy 2007, 2221(1):111-20.

[16] C. S. Ferreira, G. van Kuik, G. van Bussel, F. Scarano, "Visualization by PIV of dynamic stall on a vertical axis wind turbine", Experiments in Fluids 2008; 46(1):97-108.

[17] C.J.S. Ferreira, G. Bussel, F. Scarano, G. Kuik, "PIV visualization of dynamic stall VAWT and blade load determination", In Proc. 46th AIAA, Reno, Nevada, USA, 7-10 Jan. 2008.
[18] C.J.S. Ferreira, A. Zuijlen, H. Bijl, G. Bussel, and G. van Kuik. "Simulating dynamic stall in a two-dimensional vertical-axis wind turbine: verification and validation with particle image velocity data", Wind Energy 2010; 13(1):1-17.

[19] M. Hepperle, "JavaFoil - Analisys of Airfoils". www.mhaerotools.de.

[20] R. Eppler, D. Somers. "A Computer Program for the Design and Analysis of Low-Speed Airfoils", NASA TM80210,1980

[21] R. Bourguet, G. Martinat, G. Harran, and M. Braza, "Aerodynamic multi-criteria shape optimization of VAWT blade profile by viscous approach", Wind Energy 2007:215-19. 